OWE-018 GENERATING BETTER INFORMATION TO INFORM SERVICES FOR ALCOHOL-RELATED LIVER DISEASE: THE CONNECTED HEALTH CITIES PROGRAMME

${ }^{1}$ Constantinos Kallis*, ${ }^{1}$ Pete Dixon, ${ }^{1}$ Mustafa Shawihdi, ${ }^{1}$ Anna Jenkins, ${ }^{1}$ Benjamin Silberberg, 'Katharine Abba, 'Rabeea'h Aslam, 'Anthony Marson, ${ }^{1}$ Mike Pearson, ${ }^{1,2}$ Steve Hood, ${ }^{1,2}$ Keith Bodger. 'University of Liverpool, Liverpool, UK; ${ }^{2}$ Aintree University Hospital, Liverpool, UK

\subsection{6/gutjnl-2018-BSGAbstracts.211}

Introduction The rising burden and high mortality for alcohol-related liver disease (ARLD) is well-recognised in the UK. Efforts to plan adequate liver and alcohol services and to identify unwarranted variation are limited by lack of robust, actionable information about true workload and real-world risk-adjusted outcomes. We report an informatics programme (Connected Health Cities, Northwest Coast) that is generating new analytical resources to support services.

Methods A healthcare data lab. team of data scientists is linked to a secure 'data ark' hosting regional commissioning datasets (Admitted Patient Care, A and E, Outpatients, fiscal years $14 / 15$ to $16 / 17$ ) and is working with clinical teams to construct novel ways to interrogate data ('algorithmic approach'), model outcomes and unexplained variation and visualise data (small area mapping). Entire patient journeys were linked, to identify ARLD cases and their all-cause emergency admissions (categorised by diagnosis), capture phenotyping flags (e.g. first occurrence of codes for varices), pre-admission events and outcomes (e.g. in-hospital mortality; all-cause 30 day readmission). Maps were used to communicate catchment areas and identify admission 'hot spots'. Site-level benchmarking reports were shared with teams at 7 hospitals.

Results Compared to the standard approach for capturing cases (i.e. primary diagnosis at discharge; 6 specific ICD-10 codes; 'ARLD-Primary' Method), the algorithmic approach (ARLD-Alg) identified 9 other patterns of primary and secondary diagnoses with $>60$ primary ICD-10 codes consistent with emergency care for ARLD. Activity: Across 7 NHS Trusts, estimates of total 3 year activity for ARLD increased from 3185 (ARLD-Primary) to 5912 (ARLD-Alg) for admissions, 35840 to 56010 for bed days (equivalent to 50 extra in-patient beds), and 485 to 665 for in-hospital deaths. Casemix for the 2 approaches was similar (mean age: 51.6 v 51.4; males 63.2 v 63.6\%; Charlson Index: 1.6 v 1.7) but ARLDAlg captured more shorter stays (median LoS: 7 v 6 days; $\%$ short stay [0-2 days]: $12.1 \mathrm{v}$ 16.5). Varices cases account for $15 \%-20 \%$.

Outcomes Crude mortality across hospitals varied significantly by method ARLD-Primary: 13.0\%-21.7\%; ARLD-Alg: $10.2 \%-$ $14.5 \%$. All binary logistic models identified male patients were at lower risk of in-hospital death than females (Adj OR: 0.70). Treatment provider was a predictor of outcome.

Conclusions Standard approaches to analysis of administrative data under-estimate the burden of unplanned care for ARLD (by up to 60\%) and have dubious value for service planning or benchmarking. More sophisticated clinically-driven Methods can leverage greater value from routine data. These novel tools are scalable for nationwide deployment and have potential to inform policy and support data-driven service improvement.

Funding Department of Health

\section{OWE-035 AMBULATORY LIVER SERVICES AVOID ADMISSIONS AND REDUCE LENGTH OF STAY WITH HIGH PATIENT SATISFACTION}

J Jennings*, J Dillon, C Chimakurthi, S Gibson, A Crosswaite, S Harding, C Roberts, T Hiles, P McSorley, S Murdoch, RL Jones. Leeds Teaching Hospitals NHS Trust, Leeds, UK

\subsection{6/gutjnl-2018-BSGAbstracts.212}

Introduction Inpatient bed pressures in the NHS mean that ambulatory service development is needed. Day case and short stay units developed for elective surgery in our trust were not suited to the needs of patients with liver disease. Services managed through semi-acute pathways Resulted in unpredictable waiting times, unplanned admissions and poor patient experience. Following a pilot project in 2016-17, we describe the 2 nd phase in implementing ambulatory care services utilising a re-commissioned 4 bed ward bay in a large liver centre. Primary aims were admission avoidance (AA) and inpatient bed day savings. Secondary aims were to achieve $>70 \%$ occupancy, deliver excellent patient experience, provide facilities for earlier discharge (ED).

Methods We identified initial criteria for service delivery through a specialist nurse led unit. Patient episodes were coded to identify procedures, infusion treatments and AA/ED attendances. Safety was assessed by procedure complication rates and patient readmission rates. We used Survey Monkey to assess patient experience. Bed savings were identified from historical length of stay data in 2015-16.

Results Between 1 st May-31 st Dec 2017 there were 705 attendances. Of these: 371 large volume paracentesis, 49 urgent liver biopsy, 80 infusions, 46 ascites follow-up and 159 $\mathrm{AA} / \mathrm{ED}$ indications. Based on a 2.5 day admission for paracentesis, 927 inpatient bed days were saved for this indication and at least 420 urgent, semi-acute or unplanned admissions were avoided. Utilisation increased from 68 attendances/month to 115 in Dec 2017. The unit reached 70\% occupancy at 3 months. By Dec 2017 occupancy was >90\%. There were 3 readmissions and 1procedure complication. 95\% of patients thought that explanations regarding procedure were very clear and $95 \%$ that they were well informed throughout the day. 95\% would recommend the service to friends and family.

Conclusion A clear benefit to patients and the service was seen during the first 6 months of opening this unit. We continue to identify indications for use. Other benefits include a growing list of clear admission avoidance/early discharge for other indications. Bed day release has helped patient flow for admissions. Costs incurred in developing a specialist nursing team are offset by their other roles and transfer of ward nursing costs. Unpredicted benefits include a contribution to an overall improvement in ward quality metrics, and release of junior doctor time to direct inpatient management.

\section{ADWE-04 RIBAVIRIN FOR TREATMENT OF CHRONIC HEPATITIS E IN TRANSPLANT RECIPIENTS - EXPERIENCE FROM OUR CENTRE}

Alexander Boyd*, Sally Bufton, David Mutimer Mutimer, Ahmed Elsharkawy. Queen Elizabeth Hospital, Birmingham, UK

\subsection{6/gutjnl-2018-BSGAbstracts.213}

Introduction Hepatitis E infection usually causes self-limiting hepatitis in immunocompetent patients. In patients who have 